\title{
sciendo
}

\author{
BULGARIAN ACADEMY OF SCIENCES
}

CYBERNETICS AND INFORMATION TECHNOLOGIES • Volume 18, No 2

Sofia $\bullet 2018 \quad$ Print ISSN: 1311-9702; Online ISSN: 1314-4081

DOI: $10.2478 /$ cait-2018-0027

\section{Group Decision Analysis Algorithms with EDAS for Interval Fuzzy Sets}

Galina Ilieva

University of Plovdiv Paisii Hilendarski, 4000 Plovdiv, Bulgaria

E-mail: galili@uni-plovdiv.bg

\begin{abstract}
The purpose of this paper is proposing, analyzing and assessing two new algorithms of the EDAS method for group multi-criteria decision making with fuzzy sets. In the first proposed EDAS extension for distance measure between two interval Type-2 fuzzy numbers is applied Graded Mean Integration Representation (GMIR). The second algorithm takes into account the proximity between the fuzzy alternatives and its similarity measure is Map Distance Operator (MDO). The two new algorithms are verified by a numerical example. Comparative analysis of obtained rankings demonstrates that GMIR extension is more reliable as an interval Type-2 fuzzy alternative to Evaluation based on Distance from Average Solution (EDAS). In case that time is of the essence, the MDO EDAS could be preferred.
\end{abstract}

Keywords: Multi-criteria group decision making, fuzzy decision making, Interval Type-2 fuzzy sets, EDAS.

\section{Introduction}

The development of modern organizations can be described by uncertainty and it depends on a number of factors, related to preferred business model and surrounding environment. Vagueness that accompanies organizations' activities, impedes conducting precise experiments and leads to an inability to do exact calculations. Moreover, data necessary for making economic decisions are varied, growing rapidly and hard to extract. Therefore, rational management of a modern organization may be regarded as a multi-criteria task with inaccurate and incomplete information. As, in the general case, there is no single solution to a multi-criteria task, several different approaches for taking into account and overcoming impreciseness in management have been proposed, among them fuzzy sets [5-7, 18, 20] and fuzzy relations [14-17].

Research continues and employs more advanced forms of classic fuzzy sets. New mechanisms for crisp values calculating $[19,24]$ and new aggregation operators for summarizing relations $[4,11,22]$ are presented.

The purpose of this research is to propose and compare two Interval Type-2 Fuzzy Sets (IT2FSs) algorithms of the Evaluation based on Distance from Average Solution (EDAS) method. The rest of the paper is organized as follows: Contemporary IT2FSs decision-making applications and existing EDAS 
modifications are introduced in Section 2. Section 3 defines the basic operations for measuring distance and similarity with Interval Type-2 Fuzzy Numbers (IT2FNs) and describes the implementation steps used in fuzzy EDAS. Section 4 introduces the peculiarities in new fuzzy EDAS methods for multi-criteria group decision-making. Section 5 provides a numerical example, illustrating the proposed variants. Finally, results are compared to those obtained when applying other fuzzy EDAS and TOPSIS modifications.

\section{Literature review}

Alternatives' ranking usually requires both quantitative and qualitative evaluations. Interval Type-2 fuzzy sets are one of the approaches that help decision makers handle uncertainty and vagueness of data. A bdullah, Ad a w i y ah and Ka mal [1] have investigated a new decision making method of Interval Type-2 Fuzzy Simple Additive Weighting (IT2FSAW) as a tool in dealing with ambiguity and imprecision. The new method is applied to establish a preference in ambulance location [1].

In order to ensure more effective multi-criteria decision-making in uncertain environments, $\mathrm{Zhong}$ and $\mathrm{Y}$ a o [25] extend the elimination and choice translating reality (ELECTRE) method using interval Type-2 fuzzy numbers. They propose a $\alpha$-based distance method for measuring proximity between IT2FNs. Additionally, an entropy measure for the IT2FNs and an entropy weight model are developed to objectively determine the criteria weights without any weight information [25].

The aim of S on er, Celik and A kyuz [21] is to provide not only a hybrid theoretical methodology in multiple-attribute decision making problems, but also a practical application in maritime transportation industry. The proposed hybrid

approach integrates Analytic Hierarchy Process (AHP) method into VlseKriterijumska Optimizacijia I Kompromisno Resenje (VIKOR) technique in an Interval Type-2 Fuzzy (IT2F) environment. While AHP and VIKOR provide a comprehensive framework to solve Multiple-Attribute Decision Making (MADM) problems in maritime transportation industry, interval Type-2 fuzzy sets enables dealing with uncertainty characteristic of linguistic assessments of decision makers. Besides its robust theoretical insight, the paper has practical contribution to the naval engineers, classification societies and ship owners who have difficulty in deciding appropriate hatch cover type during construction of the ship [21].

EDAS is a relatively new method of MCDM, proposed by G h o r a b a e e et al. [10]. In EDAS, ideas from adaptive methods in MCDM TOPSIS and VIKOR have been improved. The numerous applications of the method show its potential for coping with various problems, for example, in warehouse management [9], sustainable development management [23], etc.

\section{Methodology}

The paper gives preference to interval Type-2 fuzzy sets assessments, since, on one hand, they are more flexible than classic Type-1 fuzzy sets in accounting for vagueness and uncertainty in data. On the other hand, it relies on a kind of Type-2 fuzzy sets known as interval Type-2 fuzzy numbers (IT2FNs), as it is able to deal 
more effectively with ambiguity, has better processing abilities, and simpler computations in comparison to classic Type-2 fuzzy numbers.

Suppose that $\tilde{\tilde{A}}$ and $\tilde{\tilde{B}}$ are two trapezoidal IT2FNs, where:

$$
\begin{aligned}
& \tilde{\tilde{A}}=\left(\tilde{A}^{\mathrm{T}} \mid T \in\{U, L\}\right)=\left(a_{i}^{\mathrm{T}} ; H_{1 A}^{\mathrm{T}} ; H_{2 A}^{\mathrm{T}} \mid T \in\{U, L\}, i=1,2,3,4\right), \\
& \tilde{B}=\left(\tilde{B}^{\mathrm{T}} \mid T \in\{U, L\}\right)=\left(b_{i}^{\mathrm{T}} ; H_{1 B}^{\mathrm{T}} ; H_{2 B}^{\mathrm{T}} \mid T \in\{U, L\}, i=1,2,3,4\right),
\end{aligned}
$$

and $P(\tilde{\tilde{A}})$ is a crisp number.

The basic arithmetic operations for working with IT2FNs are described in the specialized literature and we will not comment them here.

\subsection{Graded mean integration representation of IT2FNs}

Let $\left.\tilde{A}^{\mathrm{T}} \mid T \in\{U, L\}\right)=\left(a_{i}^{\mathrm{T}} ; H_{1 A}^{\mathrm{T}} ; H_{2 A}^{\mathrm{T}} \mid T \in\{U, L\}, H_{1 A}^{\mathrm{T}}=H_{2 A}^{\mathrm{T}}, i=1,2,3,4\right)$ are the upper and the lower trapezoidal membership function of $\tilde{\tilde{A}}$ respectively with the given shape function:

$$
\mu_{\tilde{A}^{\mathrm{T}}}=\left\{\begin{array}{cr}
\left(\frac{x-a_{1}^{\mathrm{T}}}{a_{2}^{\mathrm{T}}-a_{1}^{\mathrm{T}}}\right)^{n} & \text { when } x \in\left[a_{1}^{\mathrm{T}}, a_{2}^{\mathrm{T}}\right), \\
H_{1 A}^{\mathrm{T}} & \text { when } x \in\left[a_{2}^{\mathrm{T}}, a_{3}^{\mathrm{T}}\right], \\
\left(\frac{a_{4}^{\mathrm{T}}-x}{a_{4}^{\mathrm{T}}-a_{3}^{\mathrm{T}}}\right)^{n} & \text { when } x \in\left(a_{3}^{\mathrm{T}}, a_{4}^{\mathrm{T}}\right], \\
0 & \text { otherwise, }
\end{array}\right.
$$

where $n>0$. If $n=1$, then $\tilde{A}^{\mathrm{T}}$ are known as normal trapezoidal fuzzy numbers.

According to the graded mean integration representation formula [3], the crisp value of $\tilde{\tilde{A}}$ can be defined by the next equation:

$$
P(\tilde{\tilde{A}})=\frac{1}{2}\left(P\left(\tilde{A}^{\mathrm{U}}\right)+P\left(\tilde{A}^{\mathrm{L}}\right)\right)=
$$

$$
\begin{gathered}
=\frac{1}{2} \sum_{T \in\{U, L\}, p=1,2} \int_{0}^{H_{p A}^{\mathrm{T}}} h\left[\left(a_{1}^{\mathrm{T}}+\left(a_{2}^{\mathrm{T}}-a_{1}^{\mathrm{T}}\right) h^{\frac{1}{n}}+\left(a_{4}^{\mathrm{T}}-\left(a_{4}^{\mathrm{T}}-a_{3}^{\mathrm{T}}\right) h^{\frac{1}{n}}\right)\right] d h / \int_{0}^{H_{p A}^{\mathrm{T}}} h d h=\right. \\
\left.=\frac{1}{2} \sum_{T \in\{U, L\},} p=1,2 \int_{0}^{H_{p A}^{\mathrm{T}}}\left[\left(a_{1}^{\mathrm{T}}+a_{4}^{\mathrm{T}}\right) h+\left(a_{2}^{\mathrm{T}}-a_{1}^{\mathrm{T}}-a_{4}^{\mathrm{T}}+a_{3}^{\mathrm{T}}\right) h^{\frac{n+1}{n}}\right)\right] d h / \int_{0}^{H_{p A}^{\mathrm{T}}} h d h= \\
=\frac{1}{2}\left(\left(\frac{a_{1}^{\mathrm{U}}+a_{4}^{\mathrm{U}}}{2}+\frac{\left(a_{2}^{\mathrm{U}}-a_{1}^{\mathrm{U}}-a_{4}^{\mathrm{U}}+a_{3}^{\mathrm{U}}\right) H_{1 A}^{\mathrm{U}}}{3}\right)+\left(\frac{\mathrm{a}_{1}^{\mathrm{L}}+\mathrm{a}_{4}^{\mathrm{L}}}{2}+\frac{\left(a_{2}^{\mathrm{L}}-a_{1}^{\mathrm{L}}-\mathrm{a}_{4}^{\mathrm{L}}+\mathrm{a}_{3}^{\mathrm{L}}\right) H_{1 A}^{\mathrm{L}}}{3}\right)\right) .
\end{gathered}
$$

\subsection{Map distance between IT2FNs}

The degree of similarity between two interval Type- 2 trapezoidal fuzzy numbers $\tilde{\tilde{A}}$ and $\tilde{B}$ based on map distance can be determined as follows [2]:

1. Computation of the distance values $\Delta a_{i}$ and $\Delta b_{i}, i=1,2,3,4$.

In case of interval Type- 2 trapezoidal fuzzy numbers $\tilde{\tilde{A}}$ and $\tilde{\tilde{B}}$, the distance values between the upper and lower trapezoidal fuzzy numbers are calculated as follows:

$$
\Delta a_{i}=\left|a_{i}^{\mathrm{U}}-a_{i}^{\mathrm{L}}\right| \text { and } \Delta b_{i}=\left|b_{i}^{\mathrm{U}}-b_{i}^{\mathrm{L}}\right|, i=1,2,3,4 .
$$

2. Evaluation of the degree of similarity $S\left(\tilde{A}^{\Delta}, \tilde{B}^{\Delta}\right)$ between $\Delta a_{i}$ and $\Delta b_{i}$.

a) Calculation of the standard deviations $\Delta S_{a}$ and $\Delta S_{b}$ between the upper and lower fuzzy numbers:

$$
\begin{gathered}
\bar{a}^{\mathrm{U}}=\frac{1}{4}\left(a_{1}^{\mathrm{U}}+a_{2}^{\mathrm{U}}+a_{3}^{\mathrm{U}}+a_{3}^{\mathrm{U}}\right), \bar{a}^{\mathrm{L}}=\frac{1}{4}\left(a_{1}^{\mathrm{L}}+a_{2}^{\mathrm{L}}+a_{3}^{\mathrm{L}}+a_{3}^{\mathrm{L}}\right), \\
S_{\tilde{A}^{\mathrm{U}}}=\sqrt{\frac{\sum_{i=1}^{4}\left(a_{i}^{\mathrm{U}}-\bar{a}^{\mathrm{U}}\right)^{2}}{3}}, S_{\tilde{A}^{\mathrm{L}}}=\sqrt{\frac{\sum_{i=1}^{4}\left(a_{i}^{\mathrm{L}}-\bar{a}^{\mathrm{L}}\right)^{2}}{3}},
\end{gathered}
$$




$$
\Delta S_{a}=\left|S_{\tilde{A}^{\mathrm{U}}}-S_{\tilde{A}^{\mathrm{L}}}\right| .
$$

Similarly, find $\bar{b}^{\mathrm{U}}, \bar{b}^{\mathrm{L}}, S_{\tilde{B}^{\mathrm{U}}}, S_{\tilde{B}^{\mathrm{L}}}$, and $\Delta S_{b}$.

b) Determination of the map distance between the upper and lower trapezoidal fuzzy numbers:

(7) $T^{\Delta}=\frac{1}{2}\left[\left(2-\frac{1+\max \left\{\left|\Delta a_{2}-\Delta a_{1}\right|,\left|\Delta b_{2}-\Delta b_{1}\right|\right\}}{1+\min \left\{\left|\Delta a_{2}-\Delta a_{1}\right|,\left|\Delta b_{2}-\Delta b_{1}\right|\right\}}\right)+\left(2-\frac{1+\max \left\{\left|\Delta a_{4}-\Delta a_{3}\right|,\left|\Delta b_{4}-\Delta b_{3}\right|\right\}}{1+\min \left\{\left|\Delta a_{4}-\Delta a_{3}\right|,\left|\Delta b_{4}-\Delta b_{3}\right|\right\}}\right)\right]$.

c) Evaluation of the degree of similarity $S\left(\tilde{A}^{\Delta}, \tilde{B}^{\Delta}\right) \in[0,1]$ :

$$
S\left(\tilde{A}^{\Delta}, \tilde{B}^{\Delta}\right)=\left[1-\frac{\sqrt{\sum_{i=1}^{4}\left(\Delta a_{i}-\Delta b_{i}\right)^{2}}}{2}\right] \times\left[1-\sqrt{\frac{\left|\Delta S_{a}-\Delta S_{b}\right|}{2}}\right] \times\left[1-\frac{\left|H_{1 A}^{\mathrm{L}}-H_{1 B}^{\mathrm{L}}\right|}{\left|H_{1 A}^{\mathrm{U}}+H_{1 B}^{\mathrm{U}}\right|}\right] \times T^{\Delta} .
$$

3. Computing the degree of similarity $S\left(\tilde{A}^{\mathrm{U}}, \tilde{B}^{\mathrm{U}}\right)$ between $\tilde{A}^{\mathrm{U}}$ and $\tilde{B}^{\mathrm{U}}$.

a) Find the map distance between the upper trapezoidal fuzzy numbers:

$$
T^{\mathrm{U}}=\frac{1}{2}\left[\left(2-\frac{1+\max \left\{\left|a_{2}^{u}-a_{1}^{u}\right|,\left|b_{2}^{u}-b_{1}^{u}\right|\right\}}{1+\min \left\{\left|a_{2}^{u}-a_{1}^{u}\right|,\left|b_{2}^{u}-b_{1}^{u}\right|\right\}}\right)+\left(2-\frac{1+\max \left\{\left|a_{4}^{u}-a_{3}^{u}\right|,\left|b_{4}^{u}-b_{3}^{u}\right|\right\}}{1+\min \left\{\left|a_{4}^{u}-a_{3}^{u}\right|,\left|b_{4}^{u}-b_{3}^{u}\right|\right\}}\right)\right] .
$$

b) Calculate the degree of similarity $S\left(\tilde{A}^{U}, \tilde{B}^{U}\right) \in[0,1]$ :

(10) $S\left(\tilde{A}^{\mathrm{U}}, \tilde{B}^{\mathrm{U}}\right)=\left[1-\frac{\sqrt{\sum_{i=1}^{4}\left(a_{i}^{u}-b_{i}^{u}\right)^{2}}}{2}\right] \times\left[1-\sqrt{\frac{\mid S_{\widetilde{A}^{\mathrm{U}}-S_{\widetilde{B} \mathrm{U}} \mid}}{2}}\right] \times\left[\frac{\min \left(H_{1 A}^{\mathrm{U}}, H_{1 B}^{\mathrm{U}}\right)}{\max \left(H_{1 A}^{\mathrm{U}}, H_{1 B}^{\mathrm{U}}\right)}\right] \times T^{\mathrm{U}}$.

4. Evaluate the degree of similarity $S(\tilde{\tilde{A}}, \tilde{\widetilde{B}})$ between the trapezoidal fuzzy numbers $\tilde{\tilde{A}}$ and $\tilde{\tilde{B}}$ :

$$
S(\tilde{\tilde{A}}, \tilde{\tilde{B}})=\frac{S\left(\tilde{A}^{\mathrm{U}}, \tilde{B}^{\mathrm{U}}\right) \times\left(1+S\left(\tilde{A}^{\Delta}, \tilde{B}^{\Delta}\right)\right)}{2} .
$$

The greater value of $S(\tilde{\tilde{A}}, \widetilde{B})$ means that the similarity between $\tilde{\tilde{A}}$ and $\tilde{\tilde{B}}$ is greater.

\section{Proposed EDAS algorithms via IT2FSs}

Let a MCDM problem has $n$ alternatives $\left(A_{1}, \ldots, A_{n}\right)$ and $m$ decision criteria $\left(C_{1}, \ldots, C_{m}\right)$ and each alternative is assessed according to these criteria. Decision matrix $\tilde{\tilde{X}}=\left[\tilde{\tilde{x}}_{i j}\right]_{n \times m}$ shows all values which are assigned to the alternatives for each criterion. The related weight of each criterion is shown as $\widetilde{\widetilde{W}}=\left[\widetilde{\widetilde{W}}_{j}\right]_{1 \times m}$, where $\tilde{\tilde{X}}$ and $\widetilde{\widetilde{W}}$ are IT2FNs.

\subsection{EDAS modification via IT2FSs distance measure}

1. Construct the average decision matrix $\left[\tilde{\tilde{x}}_{i j}\right]_{n \times m}$ based on experts' evaluations.

2. Construct the average vector of weighted coefficients $\left[\widetilde{\widetilde{w}}_{j}\right]_{1 \times m}$.

3. Determine the average values of assessments according to criteria $\widetilde{\widetilde{A V}}_{j}$.

4. Calculate the matrices of PD $\left[\tilde{\tilde{p}}_{i j}\right]$ and ND $\left[\tilde{\tilde{n}}_{i j}\right]$ from average solution using GMIR.

5. Calculate the weighted sum of PD $\left[\widetilde{\widetilde{s p}}_{i}\right]$ and $\mathrm{ND}\left[\widetilde{\widetilde{s n}}_{i}\right]$ respectively.

6. Determine the normalized PD $\left[\widetilde{\widetilde{n p}}_{i}\right]$ and ND $\left[\widetilde{\widetilde{n n}}_{i}\right]$ for each alternative using GMIR.

7. Calculate the appraisal score $\left[{\widetilde{a s_{l}}}_{l}\right]$ for each alternative.

8. Rank the alternatives according to the appraisal score using GMIR.

Fig. 1. Flowchart of the group GMIR algorithm with IT2FSs and new distance measure 
As in classic EDAS method, in the first proposed algorithm here, a pair of fuzzy matrices is calculated - Positive Distances (PD) from the average and the Negative Distances (ND) from the average solution. The evaluation of alternatives is made according to higher average normalized values of PD and ND. The novelty here is the idea to use Graded Mean Integration Representation (GMIR) in distance calculations (Step 4, Step 6, and Step 8). The modified algorithm is given in Fig. 1.

\subsection{EDAS algorithm via IT2FSs similarity measure}

In the second proposed algorithm, in Step 3, a weighted average decision matrix is constructed. In Step 5, alternatives' proximities to the average solution are calculated. The novelty is that instead of computing the distance between fuzzy numbers, similarity measure is used, which utilizes the map distance operator (Section 3.2). Due to the symmetrical nature of the map distance formula, calculating positive and negative proximity to the average solution is not needed. The modified algorithm is presented in Fig. 2.

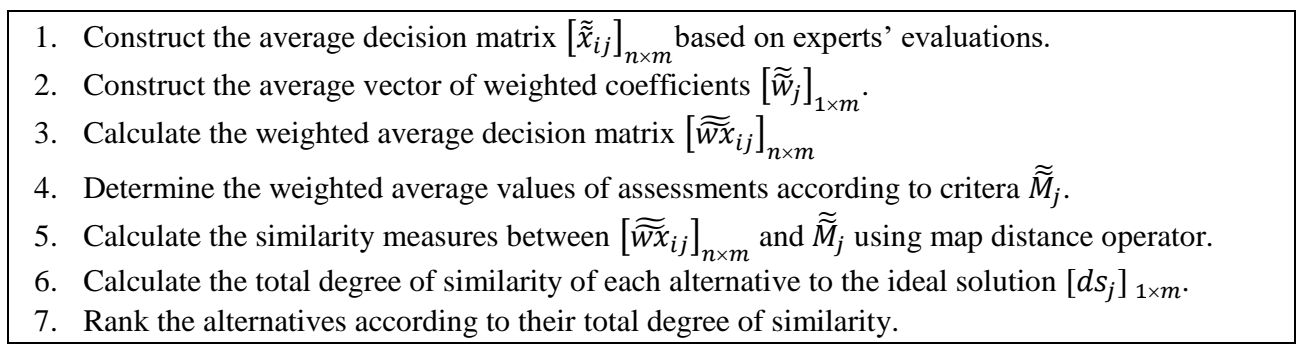

Fig. 2. Flowchart of the group MDO algorithm with IT2FSs and similarity measure section.

A detailed step-by-step description of new algorithms is provided in the next

\section{Numerical example}

Let an MCDM problem have four alternatives $\left(A_{1}, \ldots, A_{4}\right)$ and seven decision criteria $\left(C_{1}, \ldots, C_{7}\right)$, and all criteria be beneficial (maximizing). Let there be three experts, who assess the compared alternatives using IF2FNs and assign IF2FNs weights to every criterion.

Step 1. The decision matrix $\tilde{\tilde{X}}=\left[\tilde{\tilde{x}}_{i j}\right]_{4 \times 7}$ contains averaged experts' assessments of each alternative according to every criterion.

Step 2. The average relative weight of each criterion is shown in the vector $\widetilde{\widetilde{W}}=\left[\widetilde{\widetilde{w}}_{j}\right]_{1 \times 7}$.

Table 1. Decision matrix and weights of the criteria

\begin{tabular}{|c|c|c|c|c|c|c|c|}
\hline Alternative & $C_{1}$ & $C_{2}$ & $C_{3}$ & $C_{4}$ & $C_{5}$ & $C_{6}$ & $C_{7}$ \\
\hline$A_{1}$ & $\mathrm{~L}$ & $\mathrm{AH}$ & $\mathrm{VH}$ & $\mathrm{VH}$ & $\mathrm{H}$ & $\mathrm{AH}$ & $\mathrm{VH}$ \\
\hline$A_{2}$ & $\mathrm{~L}$ & $\mathrm{M}$ & $\mathrm{L}$ & $\mathrm{L}$ & $\mathrm{VL}$ & $\mathrm{AH}$ & $\mathrm{VH}$ \\
\hline$A_{3}$ & $\mathrm{M}$ & $\mathrm{M}$ & $\mathrm{L}$ & $\mathrm{H}$ & $\mathrm{L}$ & $\mathrm{M}$ & $\mathrm{H}$ \\
\hline$A_{4}$ & $\mathrm{H}$ & $\mathrm{H}$ & $\mathrm{L}$ & $\mathrm{AH}$ & $\mathrm{H}$ & $\mathrm{H}$ & $\mathrm{L}$ \\
\hline$W$ & $\mathrm{H}$ & $\mathrm{M}$ & $\mathrm{L}$ & $\mathrm{VH}$ & $\mathrm{M}$ & $\mathrm{VH}$ & $\mathrm{AH}$ \\
\hline
\end{tabular}


The values in the decision matrix and weighted coefficients are shown in Table 1 as nine grade linguistic variables. For transforming every linguistic variable into its corresponding symmetric trapezoidal IT2FN, we apply a correspondence table (Table 2). Obtained results are given in Table 3 and Table 4 in the Appendix.

Table 2. Linguistic terms and their corresponding interval type-2 fuzzy numbers

\begin{tabular}{|l|l|}
\hline Linguistic terms & Trapezoidal IT2FNs \\
\hline No influence $(\mathrm{No})$ & $((0,0,0.05,0.15 ; 1,1),(0,0,0.035,0.125 ; 0.9,0.9))$ \\
\hline Very Low (VL) & $((0,0.1,0.15,0.25 ; 1,1),(0.025,0.115,0.135,0.225 ; 0.9,0.9))$ \\
\hline Low (L) & $((0.125,0.225,0.275,0.375 ; 1,1),(0.15,0.24,0.26,0.35 ; 0.9,0.9))$ \\
\hline Medium Low (ML) & $((0.25,0.35,0.4,0.5 ; 1,1),(0.275,0.365,0.385,0.475 ; 0.9,0.9))$ \\
\hline Medium (M) & $((0.375,0.475,0.525,0.625 ; 1,1),(0.4,0.49,0.51,0.6 ; 0.9,0.9))$ \\
\hline Medium High (MH) & $((0.5,0.6,0.65,0.75 ; 1,1),(0.525,0.615,0.635,0.725 ; 0.9,0.9))$ \\
\hline High (H) & $((0.625,0.725,0.775,0.875 ; 1,1),(0.65,0.74,0.76,0.85 ; 0.9,0.9))$ \\
\hline Very High (VH) & $((0.75,0.85,0.9,1 ; 1,1),(0.775,0.865,0.885,0.975 ; 0.9,0.9))$ \\
\hline Absolutely High (AH) & $((0.875,0.975,1,1 ; 1,1),(0.9,0.99,1,1 ; 0.9,0.9))$ \\
\hline
\end{tabular}

\subsection{EDAS algorithm via IT2FNs distance measure}

Step 3. The matrix of average values of assessments according to each criterion $\widetilde{\widetilde{A V}}_{j}$ is built by using the results of Step 1 and the next equation:

$$
\widetilde{\widetilde{A V}}_{j}=\frac{\sum_{i=1}^{n} \tilde{\tilde{x}}_{i j}}{n} \text {. }
$$

Step 4. Based on Table 3, matrix of average solution from Step 3 and Equations (13) to (16), the Positive Distances (PD) and Negative Distances (ND) matrices are calculated:

$$
\begin{gathered}
\mathrm{PD}=\left[\widetilde{\widetilde{p d}}_{i j}\right]_{4 \times 7}, \\
\mathrm{ND}=\left[\widetilde{\widetilde{n d}}_{i j}\right]_{4 \times 7}, \\
\widetilde{\widetilde{p d}}_{i j}=\left\{\begin{array}{l}
\frac{\psi\left(\tilde{\tilde{x}}_{i j} \ominus \widetilde{\widetilde{a v}}_{j}\right)}{P\left(\widetilde{\widetilde{a v}}_{j}\right)} \text { if } j \in B, \\
\frac{\psi\left(\widetilde{\widetilde{a v}}_{j} \Theta \tilde{\tilde{x}}_{i j}\right)}{P\left(\widetilde{\widetilde{a v}}_{j}\right)} \text { if } j \in C,
\end{array}\right. \\
\widetilde{\widetilde{n d}}_{i j}=\left\{\begin{array}{l}
\frac{\psi\left(\widetilde{\overline{a v}}_{j} \Theta \tilde{\tilde{x}}_{i j}\right)}{P\left(\widetilde{a v}_{j}\right)} \text { if } j \in B, \\
\frac{\psi\left(\tilde{\tilde{x}}_{i j} \ominus \widetilde{\widetilde{a v}}_{j}\right)}{P\left(\widetilde{a v}_{j}\right)} \text { if } j \in C,
\end{array}\right.
\end{gathered}
$$

where function $\psi$ is defined to determine the maximum of interval Type-2 fuzzy number and zero as follows:

$$
\psi(\tilde{\tilde{A}})=\left\{\begin{array}{l}
\tilde{\tilde{A}} \text { if } P(\tilde{\tilde{A}})>0, \\
\tilde{0} \text { if } P(\tilde{\tilde{A}}) \leq 0,
\end{array}\right.
$$

where $\tilde{\tilde{0}}=((0,0,0,0 ; 1,1),(0,0,0,0 ; 1,1))$ and $B$ signifies the set of maximizing criteria, and $C$ denotes the group of minimizing criteria [9].

Steps 5-7. By using matrices of positive and negative distances and the next Equations (18)-(22), the weighted sum of positive and negative distances $\left(\widetilde{\overline{s p}_{l}}\right.$ and $\left.\widetilde{\widetilde{n n}_{l}}\right)$, their normalized values $\left(\widetilde{\widetilde{n p}}_{l}\right.$ and $\left.\widetilde{\widetilde{n n}}_{l}\right)$, and the appraisal scores are calculated for all alternatives:

$$
\widetilde{\widetilde{s p}}_{\iota}=\bigoplus_{j=1}^{m}\left(\widetilde{\widetilde{w}}_{j} \otimes \widetilde{\overline{p d}}_{i j}\right)
$$




$$
\begin{aligned}
& \widetilde{s n}_{\iota}=\bigoplus_{j=1}^{m}\left(\widetilde{\widetilde{w}}_{j} \otimes \widetilde{\widetilde{n d}}_{i j}\right) \text {, } \\
& \widetilde{\widetilde{n p}}_{l}=\frac{\widetilde{s p_{1}}}{\max _{i}\left(P\left(\widetilde{\overline{s p}_{l}}\right)\right.}, \\
& \widetilde{\widetilde{n n}}_{l}=1-\frac{\overrightarrow{s \bar{n}_{1}}}{\max _{i}\left(P\left(\widetilde{s n_{l}}\right)\right.}, \\
& \widetilde{h}_{l}=\frac{P\left(\widetilde{n n}_{i}\right)+P\left(\widetilde{n n}_{i}\right)}{?}
\end{aligned}
$$

Step 8. According to Table 6 (see Appendix), the ranking values of Appraisal Scores (AS) can be calculated. The results are as follows: $A_{1}(0.894) \succ A_{4}(0.663)>$ $A_{3}(0.318)>A_{2}(0.153)$. Therefore, $A_{1}$ is the best alternative according to the given seven criteria.

\subsection{EDAS algorithm via IT2FSs similarity measure}

As Step 1 and Step 2 are shared by both two algorithms, calculations continue from the next step:

Step 3. Calculate the weighted average decision matrix $\left[\widetilde{\widetilde{w x}}_{i j}\right]_{4 \times 7}$, where $\widetilde{\widetilde{w x}}_{i j}=\widetilde{\widetilde{\mathrm{w}}}_{j} \otimes \tilde{\tilde{x}}_{i j}, 1 \leq i \leq 4$ and $1 \leq j \leq 7$ (Table 7, see Appendix).

Step 4. Determine the weighted average values of assessments according to criteria $\left[\widetilde{\tilde{m}}_{j}\right]_{1 \times 7}$, where $\widetilde{\widetilde{m}}_{j}=\widetilde{\widetilde{w}}_{j} \otimes \widetilde{\widetilde{a v}}_{j}, 1 \leq j \leq 7$ (Table 8, see Appendix).

Step 5 and 6. Calculate the similarity measures between $\left[\widetilde{\widetilde{w x}}_{i j}\right]_{n \times m}$ and $\widetilde{\widetilde{M}}_{j}$ using the map distance operator. Results are shown in Table 9 (see Appendix), (Equations (4), (5) and (6) for $\widetilde{\widetilde{w x}}_{i j}$ ), Table 10 (see Appendix), (Equations (4), (5) and (6) for $\widetilde{\widetilde{m}}_{j}$ ) and Table 11 (see Appendix), (Equations (7), (8), (9), (10) and (11) for $\widetilde{\widetilde{w x}}_{i j}$ and $\widetilde{\widetilde{m}}_{j}$ ). After computing the total degree of similarity of each alternative to the ideal solution $\left[d s_{j}\right]_{1 \times m}$ we have: $A_{1} \approx 4.613, A_{2} \approx 4.548, A_{3} \approx 5.082, A_{4} \approx 4.465$. Final ranking is as follows: $A_{3}>A_{1}>A_{2}>A_{4}$.

\subsection{Comparison of obtained results with other heuristic EDAS methods}

In order to validate the results, we solve the same task with heuristic formulae from our literature review for determining distances between IT2FNs, such as DTraT [8] и $\subseteq$ [9]. Obtained rankings are shown in Table 3. To evaluate the qualities of the solutions obtained, we compute generalized ranking with Borda count and Copeland's methods. It turns out that the ranking given by the first variant is practically identical to the two benchmarking-rankings (for Borda count $A_{1}>A_{4}>$ $A_{3}>A_{2}$, for Copeland's method $A_{1}>A_{4}>A_{3} \approx A_{2}$ ). This is not the case with the map distance variant, however. Here, there are considerable shifts and the ranking is as follows: $A_{2}>A_{3}>A_{1}>A_{4}$ (Table 3).

Table 3. Comparing different EDAS modifications and the corresponding Borda count and Copeland's Method's (CM) ranking

\begin{tabular}{|c|c|c|c|c|c|c|c|c|}
\hline \multirow{2}{*}{$\begin{array}{c}\text { Alterna- } \\
\text { tive }\end{array}$} & \multicolumn{2}{|c|}{ EDAS GMIR } & \multicolumn{2}{c|}{ EDAS MDO } & \multicolumn{2}{c|}{ EDAS DTraT } & \multicolumn{2}{c|}{ EDAS S } \\
\cline { 2 - 9 } & Values & Rank & Values & Rank & Values & Rank & Values & Rank \\
\hline$A_{1}$ & 0.894 & 1 & 4.613 & 2 & 0.863 & 1 & 0.885 & 1 \\
\hline$A_{2}$ & 0.153 & 4 & 4.548 & 3 & 0.086 & 4 & 0.123 & 4 \\
\hline$A_{3}$ & 0.318 & 3 & 5.082 & 1 & 0.261 & 3 & 0.292 & 3 \\
\hline$A_{4}$ & 0.663 & 2 & 4.465 & 4 & 0.624 & 2 & 0.646 & 2 \\
\hline
\end{tabular}


Table 3 (c o n t in u e d)

\begin{tabular}{|c|c|c|c|c|c|c|}
\hline \multirow{2}{*}{$\begin{array}{l}\text { Alterna- } \\
\text { tive }\end{array}$} & \multicolumn{2}{|c|}{ TOPSIS DTraT } & \multicolumn{2}{|c|}{ TOPSIS GMIR } & \multirow{2}{*}{$\begin{array}{l}\text { Borda } \\
\text { count }\end{array}$} & \multirow{2}{*}{$\begin{array}{c}\mathrm{CM} \\
\text { points }\end{array}$} \\
\hline & Values & Rank & Values & Rank & & \\
\hline$A_{1}$ & 0.776 & 1 & 0.7841 & 1 & 23 & 3 \\
\hline$A_{2}$ & 0.502 & 3 & 0.4827 & 3 & 9 & 0.5 \\
\hline$A_{3}$ & 0.459 & 4 & 0.4521 & 4 & 12 & 0.5 \\
\hline$A_{4}$ & 0.521 & 2 & 0.5281 & 2 & 16 & 2 \\
\hline
\end{tabular}

Performance analysis of the two modifications shows that the mechanisms for determining similarity differ in their time complexities. The MDO method needs fewer calculations. The existence of two distance matrices, however, requires additional computations, therefore the complexity of GMIR EDAS is greater than that of MDO algorithm. For example, in the GMIR variant, two distance matrices are calculated and normalized (Equation (18) and Equation (19), respectively). Aggregating the pair of matrices is also an intensive computational process (Equations (20)-(22)). The MDO variant works with a single matrix and the number of operations is smaller (Equations (4)-(11)). Additional optimization is possible, since the average solution's characteristics (Equation (4)) are calculated only once.

Due to the fewer calculations, at first glance it may seem that MDO EDAS holds the advantage. However, this statement does not apply to algorithm's reliability, which is very important measure of new modifications' qualities from a practical point of view.

Comparative analysis with IT2FNs TOPSIS and other EDAS methods shows a deviation of MDO EDAS ranking from the average result, calculated via statistical methods. In practice, there is a possibility that deviations decrease if instead of average values calculated in situ, we work with optimal values, provided by manufacturer or experts selected in advance.

\section{Conclusions}

The paper proposes two algorithms of EDAS in a IT2FSs environment - one with GMIR defuzzification and another with MD method for measuring similarity. The advantages of the algorithms are as follows: 1) expand the applicability of EDAS in more uncertainty environments; 2) enrich the tools for distance measure in EDAS MCDM. Using a numerical example, it was proven that the new algorithms are suitable for MCDM in the absence of dependencies between assessment criteria. Obtained results were compared to results from employing existing formulae for distance between IT2FSs from specialized literature. Analysis confirms the efficiency of the two EDAS algorithms.

In the future, we plan on conducting more experiments with other fuzzy sets extensions and implementing the methods of IT2FSs MCDA in various fields of applications, such as modeling and simulating complex systems.

Acknoledgements: This research was supported by the Scientific Research Fund of the University of Plovdiv Paisii Hilendarski as a part of project SR17 FESS 012/ 24.04.2017. 


\section{References}

1. A bdu 11 a h, L., C. Ad awi y a h, C. Ka ma l. A Decision Making Method Based on Interval Type-2 Fuzzy Sets: An Approach for Ambulance Location Preference. - Applied Computing and Informatics, Vol. 14, 2018, Issue 1, pp. 65-72.

2. Che n, S.-J., Z.-Y. W a n g, W.-R. Li. Calculating the Degree of Similarity between IntervalValued Fuzzy Numbers Based on Map Distance. - In: Proc. of International Multi Conference of Engineers and Computer Scientists (IMECS'13), 2013, Hong Kong.

3. Ch e n, S. H., C. H. H s i e h. Representation, Ranking, Distance, and Similarity of L-R Type Fuzzy Number and Application. - Australia Journal of Intelligent Information Processing Systems, Vol. 6, 2000, No 4, pp. 217-229.

4. Ch i a o, K. Interval Type-2 Fuzzy Sets Multiple Criteria Decision Making Based on Quantifier Guided Aggregation Ordered Weighted Averaging Operator. - In: Proc. of Joint 17th World Congress of International Fuzzy Systems Association and 9th International Conference on Soft Computing and Intelligent Systems (IFSA-SCIS), 27-30 June 2017.

5. I 1 i e va, G. A Fuzzy Approach for Bidding Strategy Selection. - Cybernetics and Information Technologies, Vol. 12, 2012, No 1, pp. 61-69.

6. I 1 i e v a, G. Group Decision Analysis with Interval Type-2 Fuzzy Numbers. - Cybernetics and Information Technologies, Vol. 17, 2017, No 1, pp. 31-44.

7. Il i e va, G. TOPSIS Modification with Interval Type-2 Fuzzy Numbers. - Cybernetics and Information Technologies, Vol. 16, 2016, No 2, pp. 60-68.

8. K a h r a m a n, C., B. O z t a y s i, E. T u r a n o g l u. Fuzzy Analytic Hierarchy Process with Interval Type-2 Fuzzy Sets. - Knowledge-Based Systems, Vol. 59, 2014, pp. 48-57.

9. Ghor abae e, K. M., M. A miri, E. K. Zavadskas, Z. Turskis. Multi-Criteria Group Decision-Making Using an Extended EDAS Method with Interval Type-2 Fuzzy Sets. Economics and Management, Vol. 20, 2017, No 1, pp. 48-68.

10. Ghorabae e, K. M., E. K. Zavadskas, L. Olf at, Z. Turskis. Multicriteria Inventory Classification Using a New Method of Evaluation Based on Distance from Average Solution (EDAS). - Informatica, Vol. 26, 2015, No 3, pp. 435-451.

11. Li a o, H., Z. X u. Multiple Criteria Decision Making with Hesitant Fuzzy Hybrid Weighted Aggregation Operators. - In: Hesitant Fuzzy Decision Making Methodologies and Applications, Springer, 2017.

12. P e n e va, V., I. P o p ch e v. Fuzzy Criteria Importance Depending on Membership Degrees of Fuzzy Relations. - Compt. Rend. Acad. bulg. Sci., Vol. 61, 2008, No 5, pp. 579-584.

13. P e n e v a, V., I. P o p c h e v. Fuzzy Criteria Importance with Weighting Functions. - Compt. Rend. Acad. bulg. Sci., Vol. 61, 2008, No 3, pp. 293-300.

14. P e n e v a, V., I. P o p c h e v. Fuzzy Ordering on Base of Multicriteria Aggregation. - Cybernetics and Systems, an International Journal, Vol. 29, 1998, No 6, Taylor and Francis Group, Robert Trappl, Ed., pp. 613-623.

15. P e n e v a, V., I. P o p c h e v. Models for Decision Making by Fuzzy Relations and Fuzzy Numbers for Criteria Evaluations. - Compt. Rend. Acad. bulg. Sci., Vol. 62, 2009, No 10, pp. 1217-1222.

16. P e n e va, V., I. P o p che v. Multicriteria Decision Making by Fuzzy Relations and Weighting Functions for the Criteria. - Cybernetics and Information Technologies, Vol. 9, 2009, No 4, pp. 58-71.

17. P op che v, I., V. Pen eva. An Algorithm for Comparison of Fuzzy Sets. - Fuzzy Sets and Systems, Elsevier Science Publishers, North-Holland, Amsterdam, Vol. 60, 1993, No 1, pp. 59-65.

18. R a d e v a, I. Multicriteria Fuzzy Sets Application in Economic Clustering Problems. - Cybernetics and Information Technologies, Vol. 17, 2017, No 3, pp. 29-46.

19. Runkler, T., S. Coupland, J. Robert. Interval Type-2 Fuzzy Decision Making. International Journal of Approximate Reasoning, Vol. 80, 2017, pp. 217-224.

20. S h ar a f, I. TOPSIS with Similarity Measure for MADM Applied to Network Selection. Computational and Applied Mathematics, 2017, pp. 1-18. 
21. S on e r, O., E. Celik, E. A ky u z. Application of AHP and VIKOR Methods under Interval Type-2 Fuzzy Environment in Maritime Transportation. - Ocean Engineering, Vol. 129, 2017, pp. 107-116.

22. W e i, G. Pythagorean Fuzzy Interaction Aggregation Operators and Their Application to Multiple Attribute Decision Making. - Intelligent \& Fuzzy Systems, Vol. 33, 2017, No 4, pp. 2119-2132.

23. Z a va d s k a s, E. K., F. C a vall a r o, V.P odve z k o, I. U b a rt e, A. Ka k l a u s k a s. MCDM Assessment of a Healthy and Safe Built Environment According to Sustainable Development Principles: A Practical Neighborhood Approach in Vilnius. - Sustainability, Vol. 9, 2017, No $5.702 \mathrm{p}$.

24. Z h a n g, H., J. W a n g, X. C h e n. Interval Neutrosophic Sets and Their Application in Multicriteria Decision Making Problems. - Hindawi the Scientific World Journal, Vol. 2014, Article ID 645953. $15 \mathrm{p}$.

25. Z h o n g, L., L. Y a o. An ELECTRE I-Based Multi-Criteria Group Decision Making Method with Interval Type-2 Fuzzy Numbers and its Application to Supplier Selection. - Applied Soft Computing, Vol. 57, 2017, pp. 556-576.

\section{Appendix. Step-by-step calculations}

Table 4 . The average decision matrix $\left[\tilde{\tilde{x}}_{i j}\right]_{4 \times 7}$

\begin{tabular}{|c|c|c|c|c|c|c|c|c|}
\hline \multirow{2}{*}{$\tilde{\tilde{x}}_{i j}$} & \multicolumn{4}{|c|}{$\tilde{x}_{i j}^{\mathrm{U}}$} & \multicolumn{4}{|c|}{$\tilde{x}_{i j}^{\mathrm{L}}$} \\
\hline & $x_{1}^{\mathrm{U}}$ & $x_{2}^{\mathrm{U}}$ & $x_{3}^{\mathrm{U}}$ & $x_{4}^{\mathrm{U}}$ & $x_{1}^{\mathrm{L}}$ & $x_{2}^{\mathrm{L}}$ & $x_{3}^{\mathrm{L}}$ & $x_{4}^{\mathrm{L}}$ \\
\hline$\tilde{\tilde{x}}_{11}$ & 0.125 & 0.225 & 0.275 & 0.375 & 0.150 & 0.240 & 0.260 & 0.350 \\
\hline$\tilde{\tilde{x}}_{21}$ & 0.125 & 0.225 & 0.275 & 0.375 & 0.150 & 0.240 & 0.260 & 0.350 \\
\hline$\tilde{\tilde{x}}_{31}$ & 0.375 & 0.475 & 0.525 & 0.625 & 0.400 & 490 & 0.510 & 0.600 \\
\hline$\tilde{\tilde{x}}_{41}$ & 0.625 & 0.725 & 0.775 & 0.875 & 0.650 & 0.740 & 0.760 & 0.850 \\
\hline$\tilde{\tilde{x}}_{12}$ & 0.875 & 0.975 & 1.000 & 1.000 & 0.900 & 0.990 & 1.000 & 1.000 \\
\hline$\tilde{\tilde{x}}_{22}$ & 0.375 & 0.475 & 0.525 & 0.625 & 0.400 & 490 & 0.510 & .600 \\
\hline$\tilde{\tilde{x}}_{32}$ & 0.375 & 0.475 & 0.525 & 0.625 & 0.400 & 0.490 & 0.510 & 0.600 \\
\hline$\tilde{\tilde{x}}_{42}$ & 0.625 & 0.725 & 0.775 & 0.875 & 0.650 & .740 & 0.760 & 0.850 \\
\hline$\tilde{\tilde{x}}_{13}$ & 0.750 & 0.850 & 0.900 & 1.000 & 0.775 & 0.865 & 0.885 & 0.975 \\
\hline$\tilde{\tilde{x}}_{23}$ & 0.125 & 0.225 & 0.275 & 0.375 & 0.150 & 0.240 & 0.260 & 0.350 \\
\hline$\tilde{\tilde{x}}_{33}$ & 0.125 & & 0.275 & & 0.150 & 240 & 0.260 & 350 \\
\hline$\tilde{\tilde{x}}_{43}$ & 0.125 & 0.225 & 0.275 & 0.375 & 0.150 & 0.240 & 0.260 & 0.350 \\
\hline$\tilde{\tilde{x}}_{14}$ & 0.750 & 0.850 & 0.900 & 1.000 & 0.775 & 0.865 & 0.885 & 0.975 \\
\hline$\tilde{\tilde{x}}_{24}$ & 0.125 & 0.225 & 0.275 & 0.375 & 0.150 & 0.240 & 0.260 & 0.350 \\
\hline$\tilde{\tilde{x}}_{34}$ & 0.625 & 0.725 & 0.775 & 0.875 & 0.650 & 0.740 & 0.760 & 0.850 \\
\hline$\tilde{\tilde{x}}_{44}$ & 0.875 & 0.975 & 1.000 & 1.000 & 0.900 & 0.990 & 1.000 & 1.000 \\
\hline$\tilde{\tilde{x}}_{15}$ & 0.625 & 0.725 & 0.775 & 0.875 & 0.650 & 0.740 & 0.760 & 0.850 \\
\hline$\tilde{\tilde{x}}_{25}$ & 0.000 & 0.100 & 0.150 & 0. & 0.025 & 0.115 & 0.135 & 0.225 \\
\hline$\tilde{\tilde{x}}_{35}$ & 0.125 & 0. & 0.275 & & 50 & 240 & 60 & 0.350 \\
\hline$\tilde{\tilde{x}}_{45}$ & 0.625 & 0.725 & 0.775 & 0.875 & 0.650 & 0.740 & 0.760 & 0.850 \\
\hline$\tilde{\tilde{x}}_{16}$ & 0.875 & 0.975 & 1.000 & 1.000 & 0.900 & 0.990 & 1.000 & 1.000 \\
\hline$\tilde{\tilde{x}}_{26}$ & 0.875 & 0.975 & 1.000 & 1.000 & 0.900 & 0.990 & 1.000 & 1.000 \\
\hline$\tilde{\tilde{x}}_{36}$ & 0.375 & 0.475 & 0.525 & 0.625 & 0.400 & 0.490 & 0.510 & 0.600 \\
\hline$\tilde{\tilde{x}}_{46}$ & 0.625 & 0.725 & 0.775 & 0.875 & 0.650 & 0.740 & 0.760 & 0.850 \\
\hline$\tilde{\tilde{x}}_{17}$ & 0.750 & 0.850 & 0.900 & 1.000 & 0.775 & 0.865 & 0.885 & 0.975 \\
\hline$\tilde{\tilde{x}}_{27}$ & 0.750 & 0.850 & 0.900 & 1.000 & 0.775 & 0.865 & 0.885 & 0.975 \\
\hline$\tilde{\tilde{x}}_{37}$ & 0.625 & 0.725 & 0.775 & 0.875 & 0.650 & 0.740 & 0.760 & 0.850 \\
\hline$\tilde{\tilde{x}}_{47}$ & 0.125 & 0.225 & 0.275 & 0.375 & 0.150 & 0.240 & 0.260 & 0.350 \\
\hline
\end{tabular}


Table 5. The average weights' coefficients $\left[\widetilde{\widetilde{w}}_{j}\right]_{1 \times 7}$

\begin{tabular}{|c|c|c|c|c|c|c|c|c|}
\hline \multirow{2}{*}{$\widetilde{\widetilde{w}}_{j}$} & \multicolumn{4}{|c|}{$\widetilde{w}_{i j}^{\mathrm{U}}$} & \multicolumn{4}{|c|}{$\widetilde{w}_{i j}^{\mathrm{L}}$} \\
\hline & $w_{1}^{\mathrm{U}}$ & $w_{2}^{\mathrm{U}}$ & $w_{3}^{\mathrm{U}}$ & $w_{4}^{\mathrm{U}}$ & $w_{1}^{\mathrm{L}}$ & $w_{2}^{\mathrm{L}}$ & $w_{3}^{\mathrm{L}}$ & $w_{4}^{\mathrm{L}}$ \\
\hline$\widetilde{\widetilde{w}}_{1}$ & 0.625 & 0.725 & 0.775 & 0.875 & 0.650 & 0.740 & 0.760 & 0.850 \\
\hline$\widetilde{\widetilde{w}}_{2}$ & 0.375 & 0.475 & 0.525 & 0.625 & 0.400 & 0.490 & 0.510 & 0.600 \\
\hline$\widetilde{\widetilde{w}}_{3}$ & 0.125 & 0.225 & 0.275 & 0.375 & 0.150 & 0.240 & 0.260 & 0.350 \\
\hline$\widetilde{\widetilde{w}}_{4}$ & 0.750 & 0.850 & 0.900 & 1.000 & 0.775 & 0.865 & 0.885 & 0.975 \\
\hline$\widetilde{\widetilde{w}}_{5}$ & 0.375 & 0.475 & 0.525 & 0.625 & 0.400 & 0.490 & 0.510 & 0.600 \\
\hline$\widetilde{\widetilde{w}}_{6}$ & 0.750 & 0.850 & 0.900 & 1.000 & 0.775 & 0.865 & 0.885 & 0.975 \\
\hline$\widetilde{\widetilde{w}}_{7}$ & 0.875 & 0.975 & 1.000 & 1.000 & 0.900 & 0.990 & 1.000 & 1.000 \\
\hline
\end{tabular}

Table 6. The weighted sum of distances from the average solution, their normalized values and corresponding appraisal scores

\begin{tabular}{|c|c|c|c|c|c|c|c|c|}
\hline \multirow{2}{*}{$\widetilde{\widetilde{s p}}_{i}$} & \multicolumn{4}{|c|}{$\tilde{k}_{i j}^{\mathrm{U}}$} & \multicolumn{4}{|c|}{$\tilde{k}_{i j}^{\mathrm{L}}$} \\
\hline & $k_{1}^{\mathrm{U}}$ & $k_{2}^{\mathrm{U}}$ & $k_{3}^{\mathrm{U}}$ & $k_{4}^{\mathrm{U}}$ & $k_{1}^{\mathrm{L}}$ & $k_{2}^{\mathrm{L}}$ & $k_{3}^{\mathrm{L}}$ & $k_{4}^{\mathrm{L}}$ \\
\hline$\widetilde{\widetilde{s p}}_{1}$ & -0.035 & 1.142 & 1.828 & 3.371 & 0.242 & 1.347 & 1.622 & 2.958 \\
\hline$\widetilde{\widetilde{s p}}_{2}$ & -0.091 & 0.354 & 0.585 & 1.027 & 0.018 & 0.428 & 0.520 & 0.914 \\
\hline$\widetilde{\widetilde{s p}}_{3}$ & -0.911 & 0.023 & 0.466 & 1.474 & -0.664 & 0.150 & 0.327 & 1.208 \\
\hline$s \tilde{\tilde{p}}_{4}$ & 0.037 & 0.966 & 1.487 & 2.691 & 0.253 & 1.121 & 1.329 & 2.365 \\
\hline$\widetilde{\widetilde{s n}}_{1}$ & -0.078 & 0.142 & 0.262 & 0.546 & -0.015 & 0.177 & 0.225 & 0.470 \\
\hline$\widetilde{\widetilde{s n}}_{2}$ & 0.023 & 0.736 & 1.140 & 2.108 & 0.211 & 0.903 & 1.076 & 1.918 \\
\hline$\widetilde{\widetilde{s n}}_{3}$ & -0.079 & 0.377 & 0.632 & 1.246 & 0.048 & 0.452 & 0.554 & 1.077 \\
\hline$\widetilde{\widetilde{s n}}_{4}$ & -0.0 & 0. & 0 & 3 & 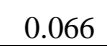 & 4 & 9 & 0.932 \\
\hline$\widetilde{\widetilde{n p}}_{1}$ & -0.023 & 0.743 & 1.188 & 2.191 & 0.157 & 0.876 & 1.054 & 1.923 \\
\hline$\widetilde{\widetilde{n p}}_{2}$ & -0.059 & 0.230 & 0.380 & 0.668 & 0.012 & 0.278 & 0.338 & 0.594 \\
\hline$\widetilde{\widetilde{n p}}_{3}$ & -0.592 & 0.015 & 0.303 & 0.959 & 0.102 & 0.097 & 0.213 & 0.786 \\
\hline$\widetilde{\widetilde{n p}}_{4}$ & 0.024 & 0.628 & 0.967 & 1.749 & 0.164 & 0.728 & 0.864 & 1.537 \\
\hline$\widetilde{\widetilde{n n}}_{1}$ & 0.454 & 0.738 & 0.858 & 1.078 & 0.530 & 0.775 & 0.823 & 1.015 \\
\hline$\widetilde{\widetilde{n n}}_{2}$ & -1.108 & -0.140 & 0.264 & 0.977 & -0.918 & -0.076 & 0.097 & 0.789 \\
\hline$\widetilde{\widetilde{n n}}_{3}$ & -0.246 & 0.368 & 0.623 & 1.079 & -0.077 & 146 & 0.548 & 0.952 \\
\hline$\widetilde{\tilde{n n}}_{4}$ & -0.053 & 0.400 & 0.612 & 1.051 & 0.068 & 0.461 & 0.546 & 0.934 \\
\hline$\tilde{\tilde{h}}_{1}$ & 0.216 & 0.740 & 1.023 & 1.635 & 0344 & 0.825 & 0.939 & 1.469 \\
\hline$\tilde{\tilde{h}}_{2}$ & -0.584 & 0.045 & 0.322 & 0.823 & $0.4 J 5$ & 0.101 & 0.218 & 0.692 \\
\hline$\tilde{\tilde{h}}_{3}$ & -0.419 & 0.191 & 0.463 & 1.019 & -0.254 & 0.272 & 0.380 & 0.869 \\
\hline$\tilde{\tilde{h}}_{4}$ & -0.014 & 0.514 & 0.789 & 1.400 & 0.116 & 0.595 & 0.705 & 1.236 \\
\hline
\end{tabular}


Table 7. The weighted average decision matrix $\left[\widetilde{\widetilde{w x}}_{i j}\right]_{4 \times 7}$

\begin{tabular}{|c|c|c|c|c|c|c|c|c|}
\hline \multirow{2}{*}{$\widetilde{\widetilde{W X}}_{i j}$} & \multicolumn{4}{|c|}{$\widetilde{w X}_{i j}^{\mathrm{U}}$} & \multicolumn{4}{|c|}{$\widetilde{w x}_{i j}^{\mathrm{L}}$} \\
\hline & $w x_{1}^{\mathrm{U}}$ & $w x_{2}^{\mathrm{U}}$ & $w x_{3}^{\mathrm{U}}$ & $w x_{4}^{\mathrm{U}}$ & $w x_{1}^{\mathrm{L}}$ & $w x_{2}^{\mathrm{L}}$ & $w x_{3}^{\mathrm{L}}$ & $w x_{4}^{\mathrm{L}}$ \\
\hline$\widetilde{\widetilde{w x}}_{11}$ & 0.078 & 0.163 & 0.213 & 0.328 & 0.098 & 0.178 & 0.198 & 0.30 \\
\hline$\widetilde{\widetilde{W X}}_{21}$ & 0.078 & 0.163 & 0.213 & 0.328 & 0.135 & 0.228 & 0.251 & 0.34 \\
\hline$\widetilde{\widetilde{W X}}_{31}$ & 0.234 & 0.344 & 0.407 & 0.547 & 0.360 & 0.466 & 0.493 & 0.59 \\
\hline$\widetilde{\widetilde{W X}}_{41}$ & 0.391 & 0.526 & 0.601 & 0.766 & 0.585 & 0.703 & 0.735 & 0.84 \\
\hline$\widetilde{\widetilde{W x}}_{12}$ & 0.328 & 0.463 & 0.525 & 0.625 & 0.360 & 0.485 & 0.510 & 0.600 \\
\hline$\widetilde{\widetilde{W x}}_{22}$ & 0.141 & 0.226 & 0.276 & 0.391 & 0.160 & 0.240 & 0.260 & 0.360 \\
\hline$\widetilde{\widetilde{W X}}_{32}$ & 0.141 & 0.226 & 0.276 & 0.391 & 0.160 & 0.240 & 0.260 & 0.360 \\
\hline$\widetilde{\widetilde{W X}}_{42}$ & 0.234 & 0.344 & 0.407 & 0.547 & 0.260 & 0.363 & 0.388 & 0.510 \\
\hline$\widetilde{\widetilde{W X}}_{13}$ & 0.094 & 0.191 & 0.248 & 0.375 & 0.116 & 0.208 & 0.230 & 0.34 \\
\hline$\widetilde{\widetilde{W X}}_{23}$ & 0.016 & 0.051 & 0.076 & 0.141 & 0.023 & 0.058 & 0.068 & 0.12 \\
\hline$\widetilde{\widetilde{w x}}_{33}$ & 0.016 & 0.051 & 0.076 & 0.141 & 0.023 & 0.058 & 0.068 & 0.12 \\
\hline$\widetilde{\widetilde{W x}}_{43}$ & 0.016 & 0.051 & 0.076 & 0.141 & 0.023 & 0.058 & 0.068 & 0.12 \\
\hline$\widetilde{\widetilde{W X}}_{14}$ & 0.563 & 0.723 & 0.810 & 1.000 & 0.601 & 0.748 & 0.783 & 0.95 \\
\hline$\widetilde{\widetilde{W x}}_{24}$ & 0.094 & 0.191 & 0.248 & 0.375 & 0.116 & 0.208 & 0.230 & 0.34 \\
\hline$\widetilde{\widetilde{W X}}_{34}$ & 0.469 & 0.616 & 0.698 & 0.875 & 0.504 & 0.640 & 0.673 & 0.83 \\
\hline$\widetilde{\widetilde{W X}}_{44}$ & 0.656 & 0.829 & 0.900 & 1.000 & 0.698 & 0.856 & 0.885 & 0.98 \\
\hline$\widetilde{\widetilde{W x}}_{15}$ & 0.234 & 0.344 & 0.407 & 0.547 & 0.260 & 0.363 & 0.388 & 0.51 \\
\hline$\widetilde{\widetilde{W x}}_{25}$ & 0.000 & 0.048 & 0.079 & 0.156 & 0.010 & 0.056 & 0.069 & 0.14 \\
\hline$\widetilde{\widetilde{W x}}_{35}$ & 0.047 & 0.107 & 0.144 & 0.234 & 0.060 & 0.118 & 0.133 & 0.21 \\
\hline$\widetilde{\widetilde{W x}}_{45}$ & 0.234 & 0.344 & 0.407 & 0.547 & 0.260 & 0.363 & 0.388 & 0.51 \\
\hline$\widetilde{\widetilde{W x}}_{16}$ & 0.656 & 0.829 & 0.900 & 1.000 & 0.698 & 0.856 & 0.885 & 0.98 \\
\hline$\widetilde{\widetilde{W x}}_{26}$ & 0.656 & 0.829 & 0.900 & 1.000 & 0.698 & 0.856 & 0.885 & 0.98 \\
\hline$\widetilde{\widetilde{W X}}_{36}$ & 0.281 & 0.404 & 0.473 & 0.625 & 0.310 & 0.424 & 0.451 & 0.59 \\
\hline$\widetilde{\widetilde{W X}}_{46}$ & 0.469 & 0.616 & 0.698 & 0.875 & 0.504 & 0.640 & 0.673 & 0.83 \\
\hline$\widetilde{\widetilde{W x}}_{17}$ & 0.656 & 0.829 & 0.900 & 1.000 & 0.698 & 0.856 & 0.885 & 0.98 \\
\hline$\widetilde{\widetilde{W X}}_{27}$ & 0.656 & 0.829 & 0.900 & 1.000 & 0.698 & 0.856 & 0.885 & 0.98 \\
\hline$\widetilde{\widetilde{w x}}_{37}$ & 0.547 & 0.707 & 0.775 & 0.875 & 0.585 & 0.733 & 0.760 & 0.85 \\
\hline$\widetilde{\widetilde{W X}}_{47}$ & 0.109 & 0.219 & 0.275 & 0.375 & 0.135 & 0.238 & 0.260 & 0.35 \\
\hline
\end{tabular}

Table 8 . The elements of the weighted average solution matrix $\left[\widetilde{\tilde{m}}_{j}\right]_{1 \times 7}$

\begin{tabular}{|l|c|c|c|c|c|c|c|c|}
\hline \multirow{2}{*}{$\widetilde{m}_{j}$} & \multicolumn{4}{|c|}{$\widetilde{M}_{j}^{\mathrm{U}}$} & \multicolumn{4}{|c|}{$\widetilde{M}_{j}^{\mathrm{L}}$} \\
\cline { 2 - 9 } & $M_{1}^{\mathrm{U}}$ & $M_{2}^{\mathrm{U}}$ & $M_{3}^{\mathrm{U}}$ & $M_{4}^{\mathrm{U}}$ & $M_{1}^{\mathrm{L}}$ & $M_{2}^{\mathrm{L}}$ & $M_{3}^{\mathrm{L}}$ & $M_{4}^{\mathrm{L}}$ \\
\hline$\widetilde{\widetilde{m}}_{1}$ & 0.195 & 0.299 & 0.358 & 0.492 & 0.219 & 0.316 & 0.340 & 0.457 \\
\hline$\widetilde{\widetilde{m}}_{2}$ & 0.211 & 0.315 & 0.371 & 0.488 & 0.235 & 0.332 & 0.354 & 0.458 \\
\hline$\widetilde{\widetilde{m}}_{3}$ & 0.035 & 0.086 & 0.119 & 0.199 & 0.046 & 0.095 & 0.108 & 0.177 \\
\hline$\widetilde{\widetilde{m}}_{4}$ & 0.445 & 0.590 & 0.664 & 0.813 & 0.480 & 0.613 & 0.643 & 0.774 \\
\hline$\widetilde{\widetilde{m}}_{5}$ & 0.129 & 0.211 & 0.259 & 0.371 & 0.148 & 0.225 & 0.244 & 0.341 \\
\hline$\widetilde{\widetilde{m}}_{6}$ & 0.516 & 0.669 & 0.743 & 0.875 & 0.552 & 0.694 & 0.723 & 0.841 \\
\hline$\widetilde{\widetilde{m}}_{7}$ & 0.492 & 0.646 & 0.713 & 0.813 & 0.529 & 0.671 & 0.698 & 0.788 \\
\hline
\end{tabular}


Table 9. The map distance calculation for matrix $\left[\widetilde{\widetilde{w x}}_{i j}\right]_{4 \times 7}$

\begin{tabular}{|c|c|c|c|c|c|}
\hline$\widetilde{\widetilde{w x}}_{i j}$ & $\overline{w x}^{\mathrm{U}}$ & $\overline{w x}^{\mathrm{L}}$ & $S_{\widetilde{W X}}{ }^{\mathrm{U}}$ & $S_{\widetilde{W X}}$ & $\Delta S_{w x}$ \\
\hline$\widetilde{\widetilde{w x}}_{11}$ & 0.196 & 0.193 & 0.104 & 0.082 & 0.022 \\
\hline$\widetilde{\widetilde{w x}}_{21}$ & 0.196 & 0.240 & 0.104 & 0.086 & 0.019 \\
\hline$\widetilde{\widetilde{w x}}_{31}$ & 0.383 & 0.477 & 0.130 & 0.095 & 0.036 \\
\hline$\widetilde{\widetilde{w x}}_{41}$ & 0.571 & 0.715 & 0.156 & 0.103 & 0.053 \\
\hline$\widetilde{\widetilde{w x}}_{12}$ & 0.485 & 0.489 & 0.124 & 0.099 & 0.025 \\
\hline$\widetilde{\widetilde{w x}}_{22}$ & 0.258 & 0.255 & 0.104 & 0.082 & 0.022 \\
\hline$\widetilde{\widetilde{w x}}_{32}$ & 0.258 & 0.255 & 0.104 & 0.082 & 0.022 \\
\hline$\widetilde{\widetilde{w x}}_{42}$ & 0.383 & 0.380 & 0.130 & 0.103 & 0.028 \\
\hline$\widetilde{\widetilde{w x}}_{13}$ & 0.227 & 0.224 & 0.117 & 0.092 & 0.025 \\
\hline$\widetilde{\widetilde{w x}}_{23}$ & 0.071 & 0.068 & 0.053 & 0.041 & 0.011 \\
\hline$\widetilde{\widetilde{w x}}_{33}$ & 0.071 & 0.068 & 0.053 & 0.041 & 0.011 \\
\hline$\widetilde{\widetilde{w x}}_{43}$ & 0.071 & 0.068 & 0.053 & 0.041 & 0.011 \\
\hline$\widetilde{\widetilde{w x}}_{14}$ & 0.774 & 0.771 & 0.182 & 0.144 & 0.039 \\
\hline$\widetilde{\widetilde{w x}}_{24}$ & 0.227 & 0.224 & 0.117 & 0.092 & 0.025 \\
\hline$\widetilde{\widetilde{w x}}_{34}$ & 0.664 & 0.661 & 0.169 & 0.133 & 0.036 \\
\hline$\widetilde{\widetilde{w x}}_{44}$ & 0.846 & 0.853 & 0.145 & 0.116 & 0.029 \\
\hline$\widetilde{\widetilde{w x}}_{15}$ & 0.383 & 0.380 & 0.130 & 0.103 & 0.028 \\
\hline$\widetilde{\widetilde{w x}}_{25}$ & 0.071 & 0.068 & 0.066 & 0.052 & 0.014 \\
\hline$\widetilde{\widetilde{w x}}_{35}$ & 0.133 & 0.130 & 0.079 & 0.062 & 0.017 \\
\hline$\widetilde{\widetilde{w x}}_{45}$ & 0.383 & 0.380 & 0.130 & 0.103 & 0.028 \\
\hline$\widetilde{\widetilde{w x}}_{16}$ & 0.846 & 0.853 & 0.145 & 0.116 & 0.029 \\
\hline$\widetilde{\widetilde{w x}}_{26}$ & 0.846 & 0.853 & 0.145 & 0.116 & 0.029 \\
\hline$\widetilde{\widetilde{w x}}_{36}$ & 0.446 & 0.443 & 0.143 & 0.113 & 0.030 \\
\hline$\widetilde{\widetilde{w x}}_{46}$ & 0.664 & 0.661 & 0.169 & 0.133 & 0.036 \\
\hline$\widetilde{\widetilde{w x}}_{17}$ & 0.846 & 0.853 & 0.145 & 0.116 & 0.029 \\
\hline$\widetilde{\widetilde{w x}}_{27}$ & 0.846 & 0.853 & 0.145 & 0.116 & 0.029 \\
\hline$\widetilde{\widetilde{w x}}_{37}$ & 0.726 & 0.732 & 0.138 & 0.110 & 0.028 \\
\hline$\widetilde{\widetilde{w x}}_{47}$ & 0.245 & 0.246 & 0.111 & 0.088 & 0.023 \\
\hline
\end{tabular}

Table 10. The map distance calculation for matrix $\left[\widetilde{\widetilde{m}}_{j}\right]_{1 \times 7}$

\begin{tabular}{|l|l|l|l|l|l|}
\hline \multicolumn{1}{|c|}{$\widetilde{\tilde{m}}_{j}$} & $\bar{m}^{\mathrm{U}}$ & $\bar{m}^{\mathrm{L}}$ & $S_{\widetilde{M}^{\mathrm{U}}}$ & $S_{\widetilde{M}^{\mathrm{L}}}$ & $\Delta S_{m}$ \\
\hline$\widetilde{\widetilde{m}}_{1}$ & 0.336 & 0.333 & 0.124 & 0.098 & 0.026 \\
\hline$\widetilde{\widetilde{m}}_{2}$ & 0.346 & 0.345 & 0.116 & 0.091 & 0.024 \\
\hline$\widetilde{\widetilde{m}}_{3}$ & 0.110 & 0.107 & 0.069 & 0.054 & 0.015 \\
\hline$\widetilde{\tilde{m}}_{4}$ & 0.628 & 0.627 & 0.153 & 0.121 & 0.032 \\
\hline$\widetilde{\tilde{m}}_{5}$ & 0.243 & 0.239 & 0.101 & 0.080 & 0.022 \\
\hline$\widetilde{\widetilde{m}}_{6}$ & 0.701 & 0.703 & 0.150 & 0.119 & 0.031 \\
\hline$\widetilde{\widetilde{m}}_{7}$ & 0.666 & 0.671 & 0.134 & 0.107 & 0.027 \\
\hline
\end{tabular}


Table 11. The map distance calculation for matrices $\left[\widetilde{\widetilde{W x}}_{i j}\right]_{4 \times 7}$ and $\left[\widetilde{\widetilde{m}}_{j}\right]_{1 \times 7}$

\begin{tabular}{|c|c|c|c|c|c|}
\hline$\widetilde{\widetilde{w x}}_{i j}$ & $T^{\Delta}$ & $S\left(\widetilde{w x}^{\Delta}, \widetilde{m}^{\Delta}\right)$ & $T^{\mathrm{U}}$ & $S\left(\widetilde{w x}^{\mathrm{U}}, \widetilde{m}^{\mathrm{U}}\right)$ & $S(\widetilde{\widetilde{W X}}, \widetilde{\widetilde{M}})$ \\
\hline$\widetilde{\widetilde{w x}}_{11}$ & 0.998 & 0.949 & 0.983 & 0.684 & 0.667 \\
\hline$\widetilde{\widetilde{w x}}_{21}$ & 0.997 & 0.905 & 0.983 & 0.684 & 0.652 \\
\hline$\widetilde{\widetilde{w x}}_{31}$ & 0.986 & 0.844 & 0.994 & 0.804 & 0.742 \\
\hline$\widetilde{\widetilde{w x}}_{41}$ & 0.972 & 0.746 & 0.972 & 0.583 & 0.509 \\
\hline$\widetilde{\widetilde{w x}}_{12}$ & 0.996 & 0.970 & 0.978 & 0.707 & 0.697 \\
\hline$\widetilde{\widetilde{w x}}_{22}$ & 0.999 & 0.964 & 0.990 & 0.752 & 0.738 \\
\hline$\widetilde{\widetilde{w x}}_{32}$ & 0.999 & 0.964 & 0.990 & 0.752 & 0.738 \\
\hline$\widetilde{\widetilde{w x}}_{42}$ & 0.998 & 0.954 & 0.987 & 0.780 & 0.762 \\
\hline$\widetilde{\widetilde{w x}}_{13}$ & 0.995 & 0.915 & 0.956 & 0.636 & 0.609 \\
\hline$\widetilde{\widetilde{w x}}_{23}$ & 0.999 & 0.954 & 0.985 & 0.774 & 0.756 \\
\hline$\widetilde{\widetilde{w x}}_{33}$ & 0.999 & 0.954 & 0.985 & 0.774 & 0.756 \\
\hline$\widetilde{\widetilde{W x}}_{43}$ & 0.999 & 0.954 & 0.985 & 0.774 & 0.756 \\
\hline$\widetilde{\widetilde{w x}}_{14}$ & 0.997 & 0.934 & 0.975 & 0.657 & 0.635 \\
\hline$\widetilde{\widetilde{w x}}_{24}$ & 0.997 & 0.930 & 0.969 & 0.452 & 0.436 \\
\hline$\widetilde{\widetilde{w x}}_{34}$ & 0.998 & 0.951 & 0.986 & 0.775 & 0.756 \\
\hline$\widetilde{\widetilde{w x}}_{44}$ & 0.995 & 0.949 & 0.966 & 0.635 & 0.619 \\
\hline$\widetilde{\widetilde{w x}}_{15}$ & 0.997 & 0.936 & 0.974 & 0.661 & 0.640 \\
\hline$\widetilde{\widetilde{w x}}_{25}$ & 0.997 & 0.929 & 0.968 & 0.623 & 0.601 \\
\hline$\widetilde{\widetilde{w x}}_{35}$ & 0.998 & 0.945 & 0.980 & 0.700 & 0.681 \\
\hline$\widetilde{\widetilde{w x}}_{45}$ & 0.997 & 0.936 & 0.974 & 0.661 & 0.640 \\
\hline$\widetilde{\widetilde{w x}}_{16}$ & 0.997 & 0.960 & 0.977 & 0.713 & 0.699 \\
\hline$\widetilde{\widetilde{w x}}_{26}$ & 0.997 & 0.960 & 0.977 & 0.713 & 0.699 \\
\hline$\widetilde{\widetilde{w X}}_{36}$ & 0.997 & 0.972 & 0.977 & 0.618 & 0.609 \\
\hline$\widetilde{\widetilde{w x}}_{46}$ & 0.997 & 0.942 & 0.977 & 0.760 & 0.737 \\
\hline$\widetilde{\widetilde{w x}}_{17}$ & 0.999 & 0.965 & 0.992 & 0.679 & 0.667 \\
\hline$\widetilde{\widetilde{w x}}_{27}$ & 0.999 & 0.965 & 0.992 & 0.679 & 0.667 \\
\hline$\widetilde{\widetilde{w x}}_{37}$ & 1.000 & 0.980 & 0.997 & 0.808 & 0.801 \\
\hline$\widetilde{\widetilde{w x}}_{47}$ & 0.998 & 0.943 & 0.980 & 0.455 & 0.442 \\
\hline
\end{tabular}

Received 22.03.2018; Accepted 05.04.2018 